\title{
Supplementary material for: A Bayesian Hierarchical Model for Criminal Investigations
}

\author{
F.O. Bunnin*† and J.Q. Smith ${ }^{* \dagger}$,
}

\begin{abstract}
This document provides appendices detailing the construction of the task probabilities conditional on position; the semi-Markov transition matrix and resulting recurrence equations; figures from the analysis of RDCEG model structure robustness; and figures for parameter sensitivity.
\end{abstract}

\section{Contents}

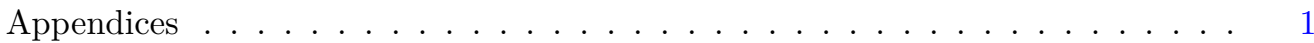

A Task probabilities given position . . . . . . . . . . . . . . 2

B The semi-Markov transition matrix and resulting recurrence equations . . . . 3

C Multiple Attack Method Scenarios . . . . . . . . . . . . . . . 5

D RDCEG robustness . . . . . . . . . . . . . . . . . 11

E Sensitivity analysis . . . . . . . . . . . . . . . . . 18

\footnotetext{
* The Alan Turing Institute, British Library, 96 Euston Road, London NW1 2DB, JSmith@turing.ac.uk OBunnin@turing.ac.uk

$\dagger$ Data Science Institute, Imperial College London, South Kensington Campus, SW7 2AZ FBunnin@imperial.sc.uk

$\ddagger$ Department of Statistics, University of Warwick, Coventry, CV4 7AL, United Kingdom, J.Q.Smith@warwick.ac.uk

(C) 0000 International Society for Bayesian Analysis

DOI: 0000
} 


\section{Appendix A: Task probabilities given position}

Some settings that keep elicitation of task probabilities to the minimum whilst appearing to provide good discriminatory power in most circumstances are given below. For each state $w_{i}, i=0,1,2, \ldots, m$, let

$$
p_{t, i}^{I^{*}\left(w_{i}\right)} \triangleq P\left\{\boldsymbol{\theta}_{I_{+}^{*}\left(w_{i}\right)}=\mathbf{1}, \boldsymbol{\theta}_{I_{-}^{*}\left(w_{i}\right)}=\mathbf{0} \mid w_{i}, \mathcal{F}_{t-}\right\}
$$

be the probability that all the positive tasks and none of the negative tasks are being done given the position is $w_{i}$. For definitions of $I^{*}\left(w_{i}\right), I_{+}^{*}\left(w_{i}\right), I_{-}^{*}\left(w_{i}\right)$ see Equations 1 in Section 3. We define and elicit tasks in such a way that

$$
p_{t, i}^{I^{*}\left(w_{i}\right)} \geq 0.2
$$

i.e. given someone is in active position $w_{i}$ then the probability that $\omega$ in $w_{i}$ is engaged in all the tasks that are positive indicators and none of the tasks that are negative indicators is non-negligible. We also assume that the less the engagement in the positive tasks the smaller the probability $\omega$ lies in this active state and the contrary for the negative tasks.

Let $r_{i}^{-}$and $r_{i}^{+}$denote the cardinality of the sets $I_{-}^{*}\left(w_{i}\right)$ and $I_{+}^{*}\left(w_{i}\right)$. Suppose $\emptyset \subseteq$ $A \subseteq I_{+}^{*}\left(w_{i}\right)$ where $0 \leq r(A) \leq r_{i}^{+}$is the cardinality of $A$ and $\emptyset \subseteq B \subseteq I_{-}^{*}\left(w_{i}\right)$ where $0 \leq r(B) \leq r_{i}^{-}$is the cardinality of $B$. Then

$$
0 \leq K(A, B) \triangleq r(A)+r_{i}^{-}-r(B) \leq r_{i}^{+}+r_{i}^{-}
$$

so $K$ is the number of tasks being done in $I_{+}^{*}\left(w_{i}\right)$ plus those not being done in $I_{-}^{*}\left(w_{i}\right)$. Let the log odds be:

$$
\phi_{t, i}^{I^{*}\left(w_{i}\right)} \triangleq \log p_{t, i}^{I^{*}\left(w_{i}\right)}-\log \left\{1-p_{t, i}^{I^{*}\left(w_{i}\right)}\right\}
$$

Then under the Naive Bayes assumption of Section 4 for $w_{0}$, we set

$$
\phi_{t, 0}^{I^{*}\left(w_{i}\right)}=\sum_{k \in I^{*}\left(w_{i}\right)} \phi_{t, 0}^{k}
$$

Then the full set of probabilities for any such $A$ and $B$ can be generated by interpolating:

$$
\begin{gathered}
\phi_{t}^{i, A, B}=\alpha_{K(A, B), i} \phi_{t, i}^{I^{*}\left(w_{i}\right)}+\left(1-\alpha_{K(A, B), i}\right) \phi_{t, 0}^{I^{*}\left(w_{i}\right)} \\
\alpha_{K(A, B), i}=\left(\frac{K(A, B)}{r_{i}^{+}+r_{i}^{-}}\right)^{\xi_{i}}
\end{gathered}
$$

where $\xi_{i}>0$ is constrained such that the resulting probabilities sum to one. This setting has the property that preserves the sorts of monotonicity we require for the comment above. 


\section{Appendix B: The semi-Markov transition matrix and resulting recurrence equations}

We now explicitly model the process $W_{t}$ as a continuous time process, still with discrete state space. We still assume that we observe data at discrete sequential times: $\left(t_{i}\right)$, $i \in \mathbb{N}$ but we model explicitly that the suspect may transition between states between or at these observation times. Thus the discrete time process $W_{t_{n}}$ is embedded in a continuous time semi-Markov process $W_{t}, t \in \mathbb{R}^{+}$.

Assume that the time interval is short so that at most one transition has occurred between $t$ and $t^{\prime}$. Conditioning on the event that there has only been one transition, let $\zeta_{i}\left(t, t^{\prime}\right)$ represent the probability of a transition from $w_{i}$ during the time interval $\left(t, t^{\prime}\right]$, where $\zeta_{i}$ is the holding distribution for the $i^{t h}$ position. Let $M_{i j}^{0}$ represent the probability that the suspect will transition to the position $w_{j}$ from position $w_{i}$ given there has been exactly one state transition. Then the components of the transition matrix are given by:

$$
M_{i j}\left(t, t^{\prime}, M_{i, j}^{0}, \zeta_{i}\right)=\left\{\begin{array}{l}
1-\zeta_{i}\left(t, t^{\prime}\right), i=j \\
\zeta_{i}\left(t, t^{\prime}\right) M_{i j}^{0}, i \neq j
\end{array}\right.
$$

Using the RDCEG of the vehicle attacker in Section 5, we have $M^{0}$ and $M\left(t, t^{\prime}\right)$ as in Table 1 with $m_{i, j}$ denoting edge probabilities: i.e. the probability of transition from the source vertex $w_{i}$ to the destination vertex $w_{j}$ conditional on a transition having occurred.

\begin{tabular}{lccccc}
\hline$M^{0}$ & Neutral & ActiveConvert & Training & Preparing & Mobilised \\
\hline $\mathrm{N}$ & 0 & 0 & 0 & 0 & 0 \\
$\mathrm{~A}$ & $m_{a, n}$ & 0 & $m_{a, t}$ & $m_{a, p}$ & 0 \\
$\mathrm{~T}$ & $m_{t, n}$ & 0 & 0 & $m_{t, p}$ & 0 \\
$\mathrm{P}$ & $m_{p, n}$ & 0 & 0 & 0 & $m_{p, m}$ \\
$\mathrm{M}$ & $m_{m, n}$ & 0 & 0 & $m_{m, p}$ & 0 \\
\hline$M\left(t, t^{\prime}\right)$ & Neutral & ActiveConvert & Training & Preparing & Mobilised \\
\hline $\mathrm{N}$ & 1 & 0 & 0 & 0 & 0 \\
$\mathrm{~A}$ & $\zeta_{a}\left(t, t^{\prime}\right) m_{a, n}$ & $1-\zeta_{a}\left(t, t^{\prime}\right)$ & $\zeta_{a}\left(t, t^{\prime}\right) m_{a, t}$ & $\zeta_{a}\left(t, t^{\prime}\right) m_{a, p}$ & 0 \\
$\mathrm{~T}$ & $\zeta_{t}\left(t, t^{\prime}\right) m_{t, n}$ & 0 & $1-\zeta_{t}\left(t, t^{\prime}\right)$ & $\zeta_{t}\left(t, t^{\prime}\right) m_{t, p}$ & 0 \\
$\mathrm{P}$ & $\zeta_{p}\left(t, t^{\prime}\right) m_{p, n}$ & 0 & 0 & $1-\zeta_{p}\left(t, t^{\prime}\right)$ & $\zeta_{p}\left(t, t^{\prime}\right) m_{p, m}$ \\
$\mathrm{M}$ & $\zeta_{m}\left(t, t^{\prime}\right) m_{m, n}$ & 0 & 0 & $\zeta_{m}\left(t, t^{\prime}\right) m_{m, p}$ & $1-\zeta_{m}\left(t, t^{\prime}\right)$ \\
\hline
\end{tabular}

Table 1: Semi-Markov Transition Matrix 
Under the assumption that the observation times are frequent enough that at most one transition could occur between them, the filtering equations to update the position probabilities given the change in time and the observed data are

$$
\begin{array}{r}
p\left(w_{i, t_{n+1}} \mid Z_{t_{n+1}}\right) \propto p\left(w_{i}, t_{n+1} \mid Z_{t_{n}}\right) \times \\
\sum_{\theta_{I^{*}\left(w_{i}\right)} \in\{0,1\}^{\left|I^{*}\left(w_{i}\right)\right|}} p\left(\theta_{I^{*}\left(w_{i}\right)} \mid w_{i}\right) p\left(Z_{I^{*}\left(w_{i}\right), t_{n+1}} \mid \theta_{I^{*}\left(w_{i}\right)}\right) \\
p\left(w_{i, t_{n+1}} \mid Z_{t_{n}}\right)=\sum_{j=0}^{d} p\left(w_{j, t_{n}} \mid Z_{t_{n}}\right) M_{j, i}\left(t_{n}, t_{n+1}, M^{0}, \zeta\right)
\end{array}
$$

Equation (5) updates the position probabilities given the observed data at time $t_{n+1}$ whilst Equation (6) transitions the position probabilities over a small time interval $\left(t_{n}, t_{n+1}\right)$ according to the transition matrix $M\left(t_{n}, t_{n+1}, M^{0}, \zeta\right)$. Now we ease the assumption that observation times are frequent enough that we can exclude the possibility of more than one state transition between them. For ease of exposition and notation we assume that the intervals between observation times are a multiple of $\left|t-t^{\prime}\right|$, ie

$$
t_{n+1}-t_{n}=k\left|t^{\prime}-t\right|
$$

for every $t_{n}$ and for some $k \in \mathbb{N}$. Here we take the holding time distributions $\zeta_{i}\left(t, t^{\prime}\right)$ as time-homogenous although in future work we plan to use inhomogenous distributions. With homogeneity the transition matrix between observation times is then simply the appropriate power of $M\left(t, t^{\prime}\right)$, i.e.

$$
M\left(t_{n}, t_{n+1}, M^{0}, \zeta\right)=M^{k}\left(t, t^{\prime}, M^{0}, \zeta\right)
$$

Then Equation (6) can be written as:

$$
p\left(w_{i, t_{n+1}} \mid Z_{t_{n}}\right)=\sum_{j=0}^{d} p\left(w_{j, t_{n}} \mid Z_{t_{n}}\right)\left(M^{k}\left(t, t^{\prime}, M^{0}, \zeta\right)\right)_{i, j}
$$

The prior and posterior log odds formulae used in Equations 8 and 10 of the main paper are amended to be based on $Z$ rather than $Y$ and to function as filtering recurrence equations based on $Z_{t}$. Suppressing $\mathcal{F}_{t-}$ to simplify the notation we obtain:

$$
\rho_{i t} \triangleq \log \left(\frac{p\left(w_{i} \mid \boldsymbol{Z}_{I^{*}\left(w_{i}\right) t_{n}}\right)}{p\left(w_{0} \mid \boldsymbol{Z}_{t_{n}}\right)}\right), \rho_{i t}^{*} \triangleq \log \left(\frac{p\left(w_{i} \mid \boldsymbol{Z}_{I^{*}\left(w_{i}\right) t_{n+1}}\right)}{p\left(w_{0} \mid \boldsymbol{Z}_{t_{n+1}}\right)}\right)
$$

The the loglikelihood ratio of $Z_{t_{n+1}}$ becomes

$$
\lambda_{i}\left(\boldsymbol{Z}_{I^{*}\left(w_{i}\right) t_{n+1}}\right) \triangleq \log \left(\frac{\sum_{\theta_{I^{*}\left(w_{i}\right)}} p\left(\boldsymbol{Z}_{I^{*}\left(w_{i}\right) t_{n+1}} \mid \theta_{I^{*}\left(w_{i}\right)}\right) p\left(\theta_{I^{*}\left(w_{i}\right)} \mid w_{i}\right)}{\sum_{\theta_{I^{*}\left(w_{i}\right)}} p\left(\boldsymbol{Z}_{I^{*}\left(w_{i}\right) t_{n+1}} \mid \theta_{I^{*}\left(w_{i}\right)}\right) p\left(\theta_{I^{*}\left(w_{i}\right)} \mid w_{0}\right)}\right)
$$

and the update formula on the log odds is

$$
\rho_{i t}^{*}=\rho_{i t}+\lambda_{i}\left(\boldsymbol{Z}_{I^{*}\left(w_{i}\right) t}\right)
$$




\section{Appendix C: Multiple Attack Method Scenarios}

These scenarios are used for the model diagnostic analyses of Appendix D and E.

- Scenario A: The suspect is seen to make radical statements both in public and privately including public threats; they increase their meetings with known radicals and other suspected cell members; they sell assets, increase their financial resources, hire a vehicle, investigate bomb-making and technical web-sites; they are seen electronically and physically visiting target locations and are then seen to be making large financial expenditures. The standardised data is displayed as stacked bar charts in Figures 1a to 3b; and the resulting task intensities are shown in Figures $4 \mathrm{a}$ to $5 \mathrm{~b}$.

- Scenario B: The suspect reduces public and private contact and engagement with radicals and at no time makes and radical statements or threats. 


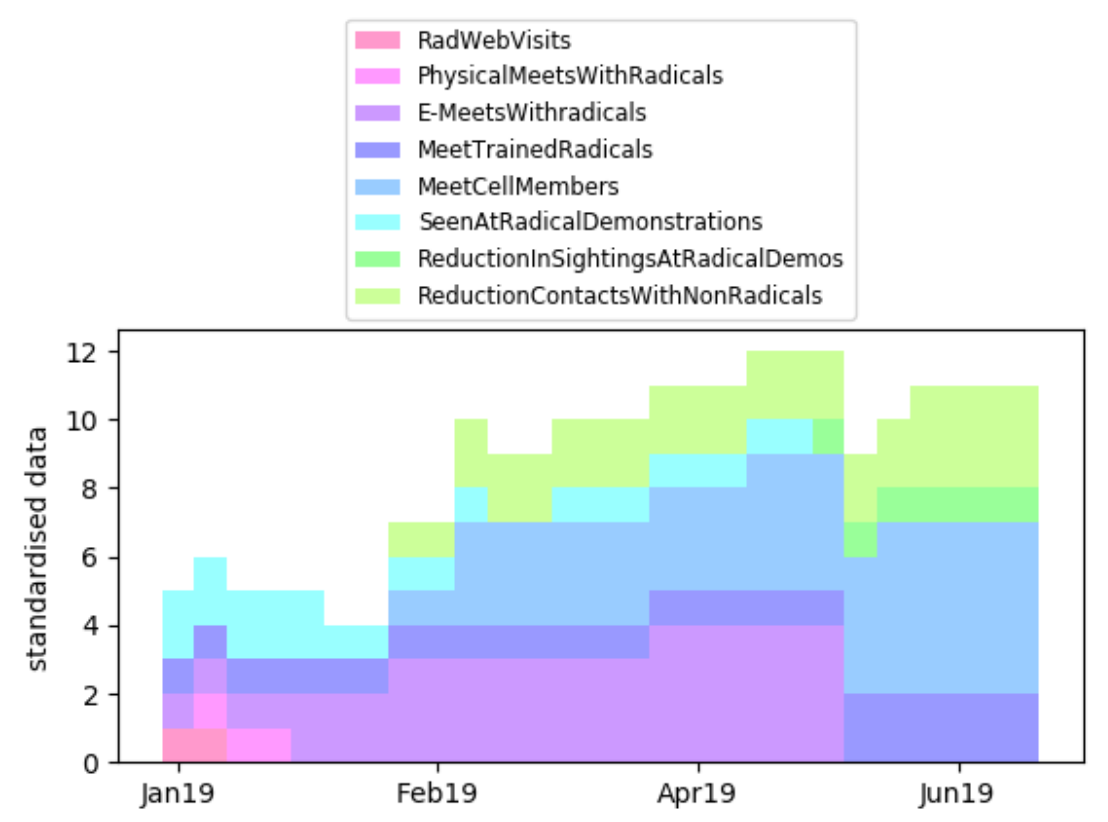

(a) General Data

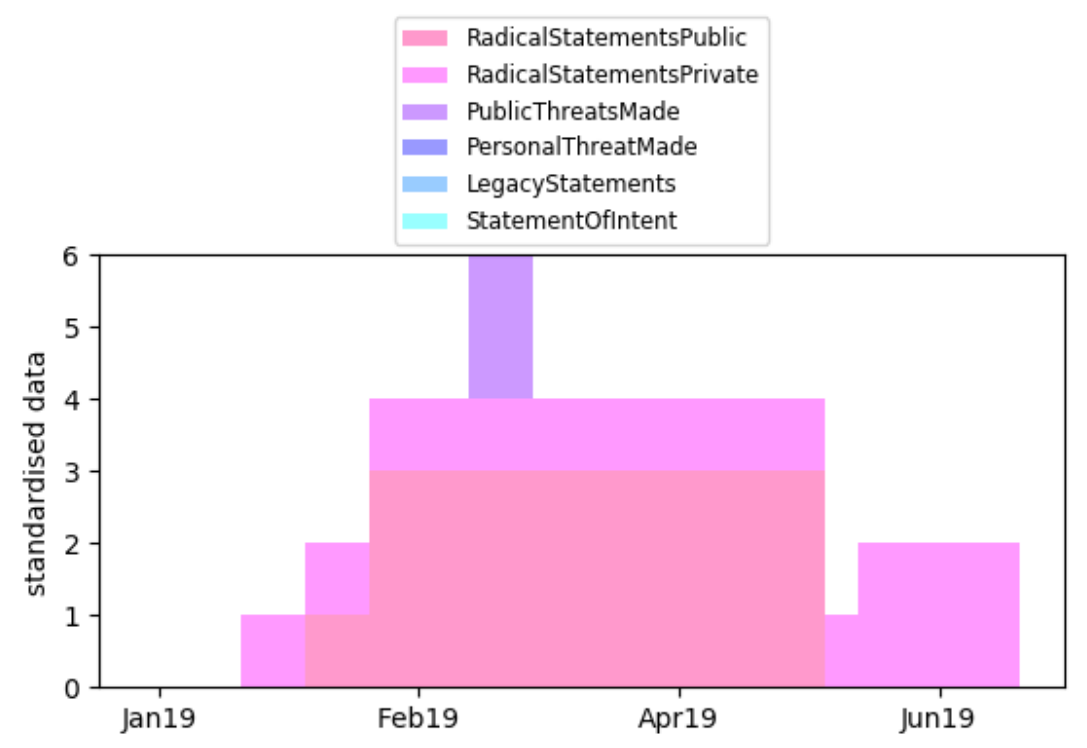

(b) Threat Statement Data

Figure 1

imsart-ba ver. 2014/10/16 file: SuppHM.tex date: December 4, 2019 


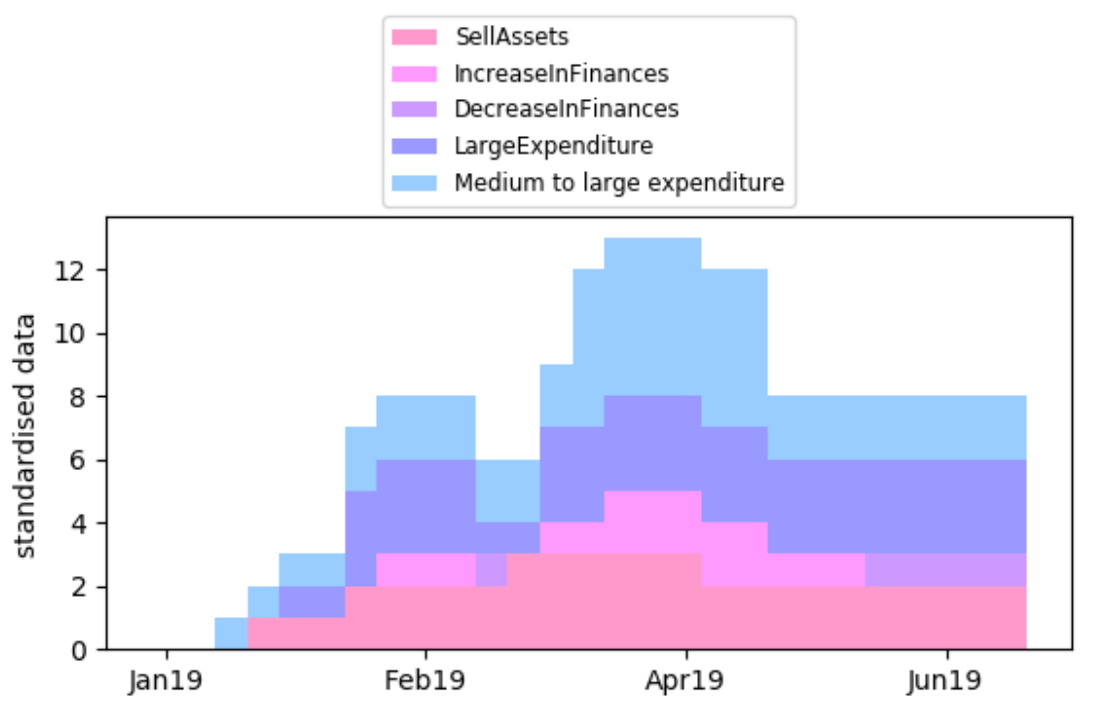

(a) Finance Data

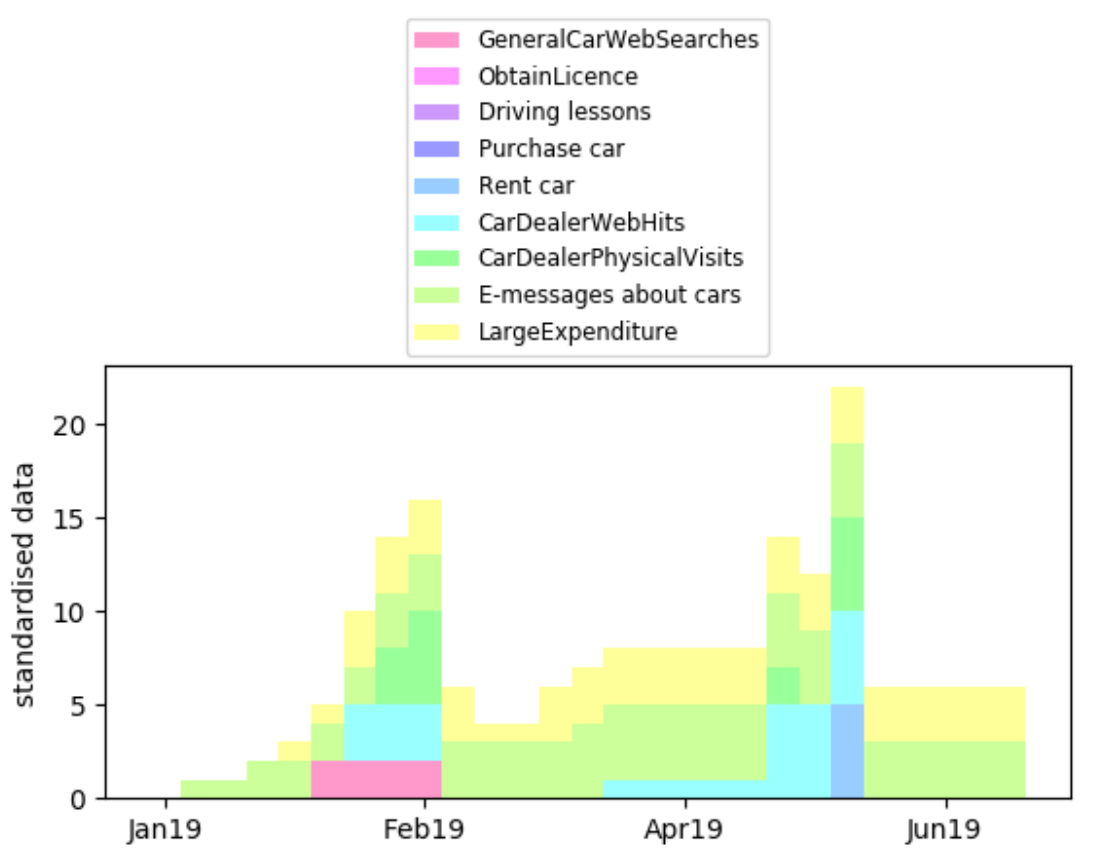

(b) Vehicle Data

Figure 2

imsart-ba ver. 2014/10/16 file: SuppHM.tex date: December 4, 2019 


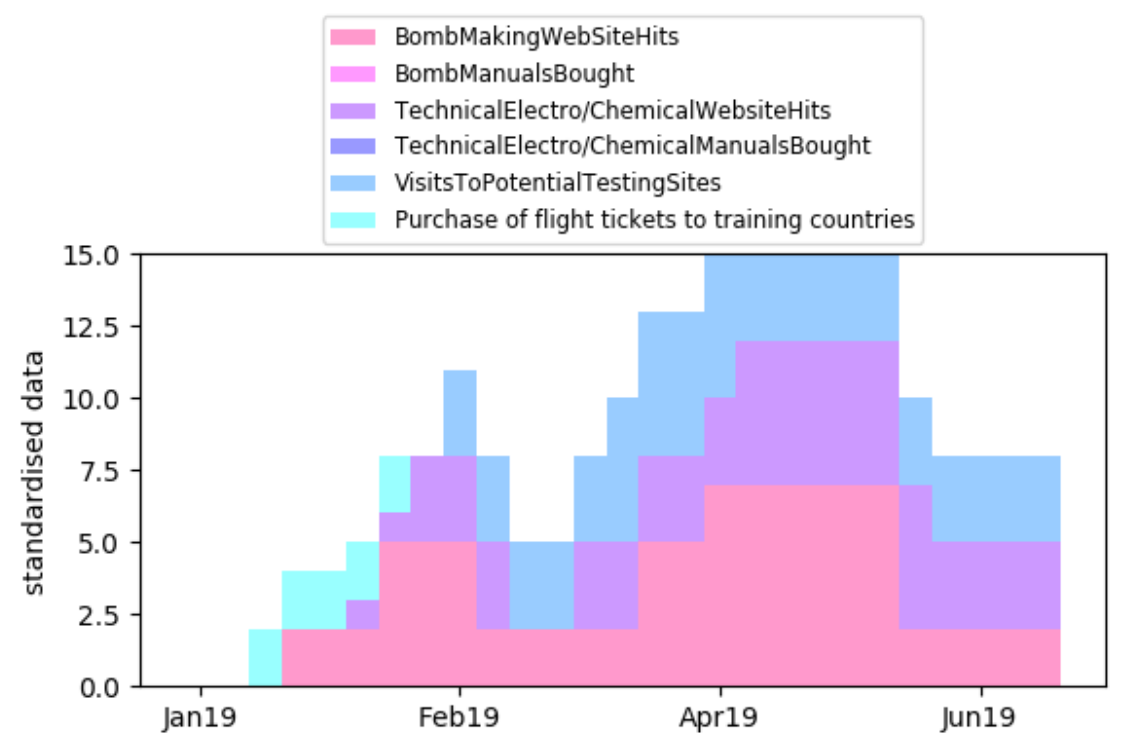

(a) Bomb Data

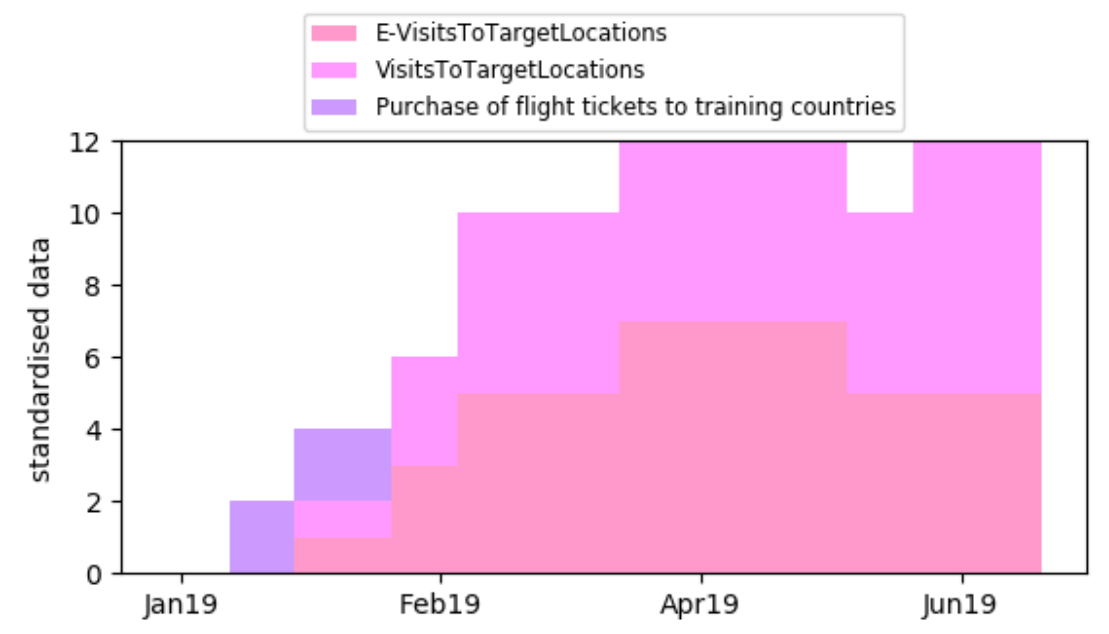

(b) Movement Data

Figure 3

imsart-ba ver. 2014/10/16 file: SuppHM.tex date: December 4, 2019 


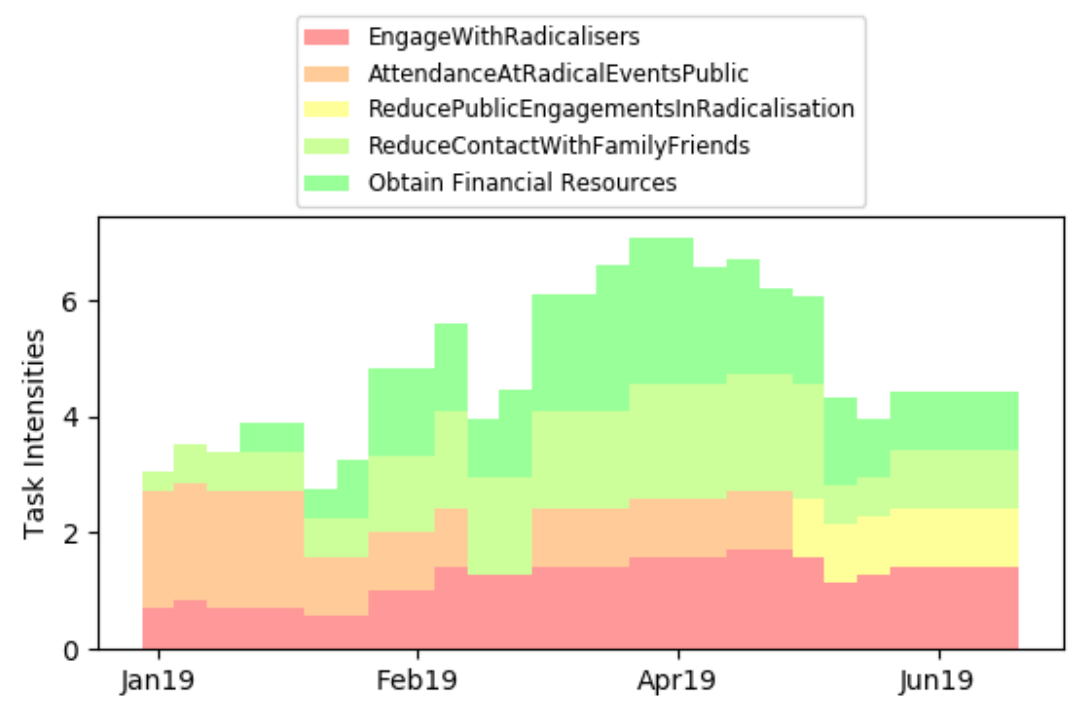

(a) General Task Intensities for scenario A

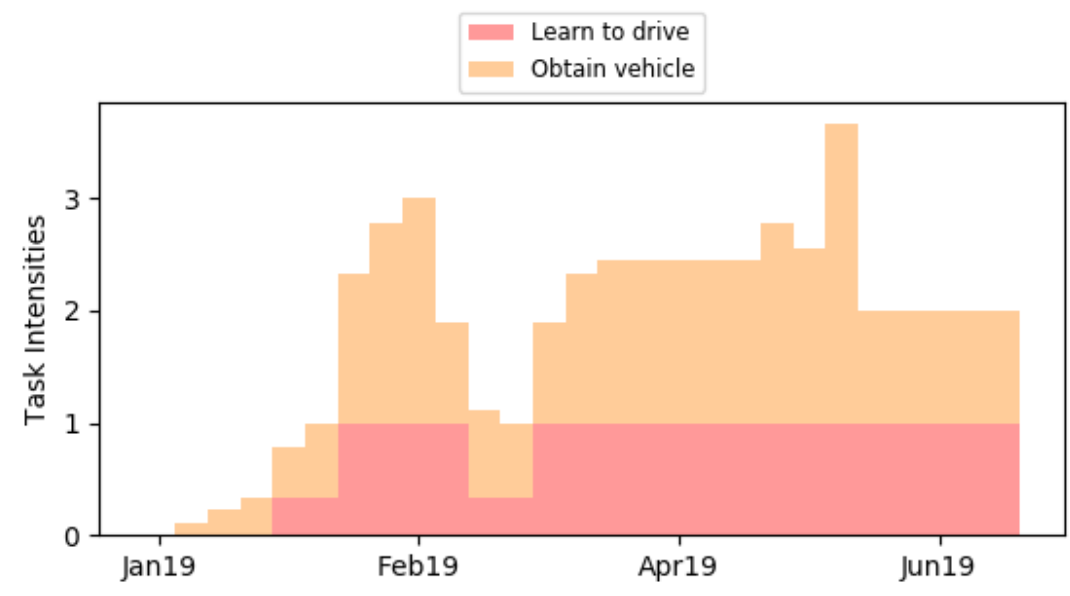

(b) Vehicle Task Intensities for scenario A

Figure 4

imsart-ba ver. 2014/10/16 file: SuppHM.tex date: December 4, 2019 


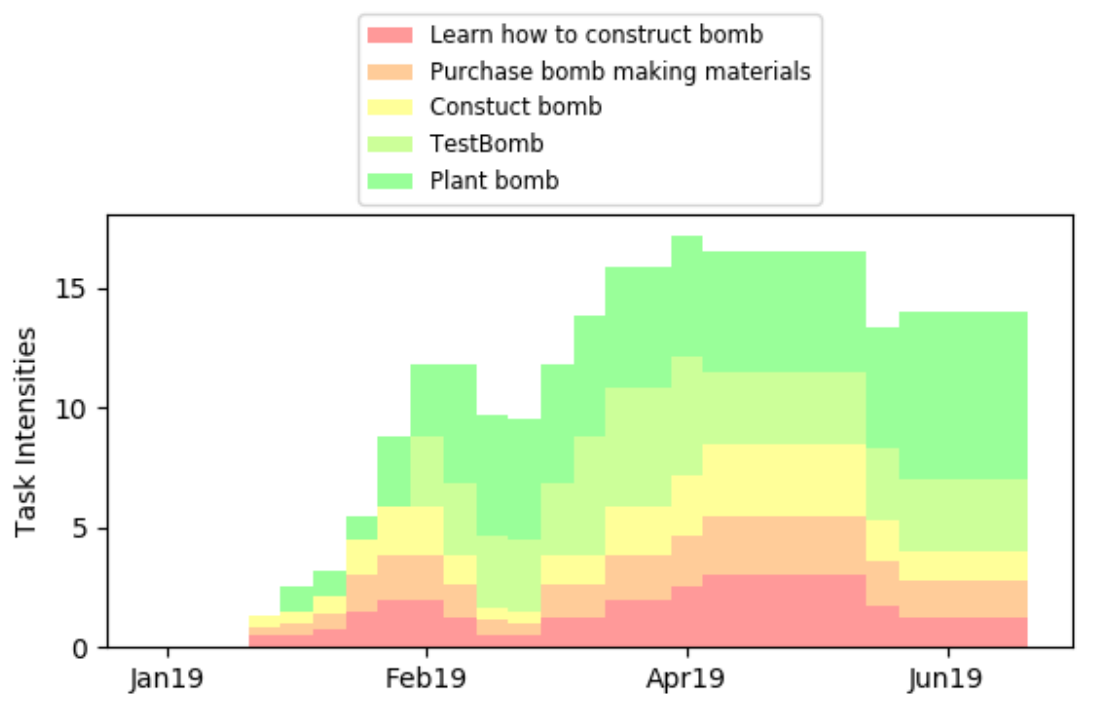

(a) Bomb Task Intensities for scenario $A$

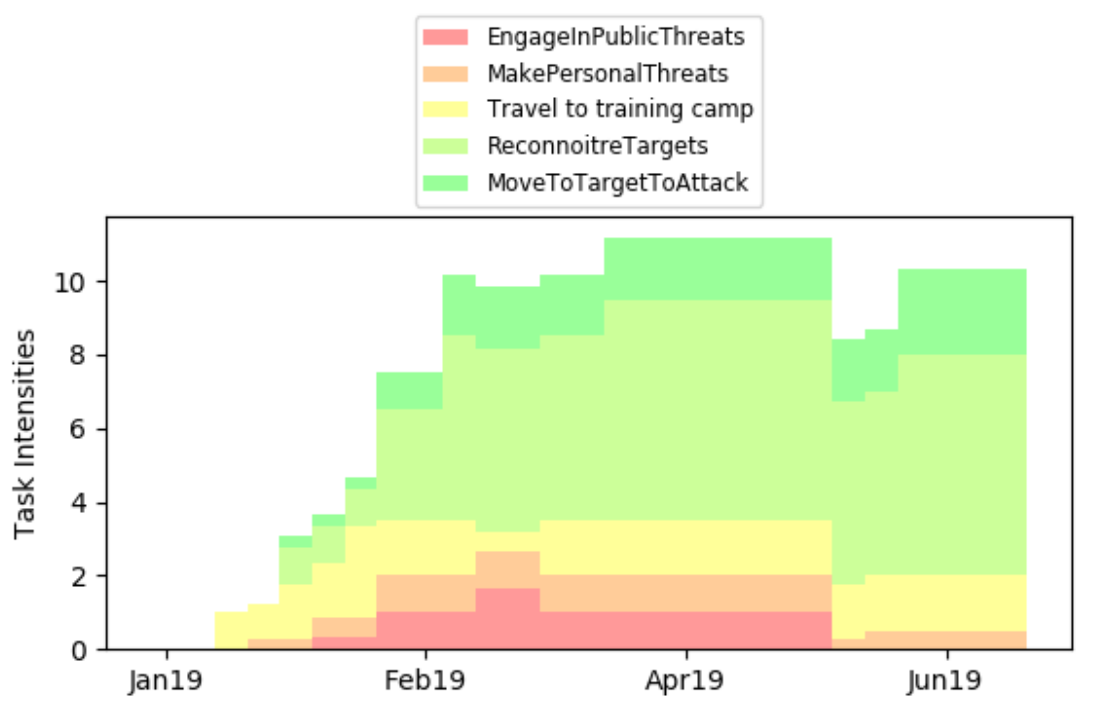

(b) Threat and Mobilisation Task Intensities for scenario A

Figure 5

imsart-ba ver. 2014/10/16 file: SuppHM.tex date: December 4, 2019 


\section{Appendix D: RDCEG robustness}

We use the model as described in Section 5 as the base RDCEG structure, base prior state probabilities and base holding distribution. We use Scenarios A and B from Appendix $\mathrm{C}$ to analyse the effect on the probability evolution through time under changes in the RDCEG structure. The results are illustrated in Figures 6 to 12

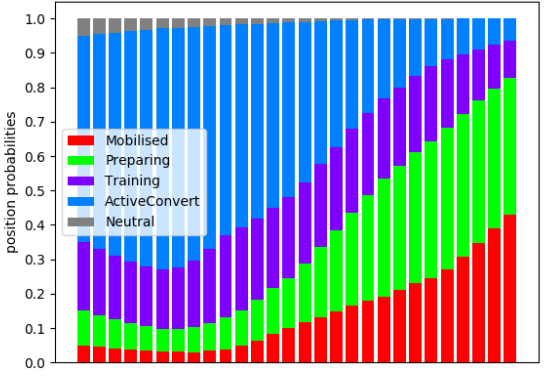

(a) State probabilities through time

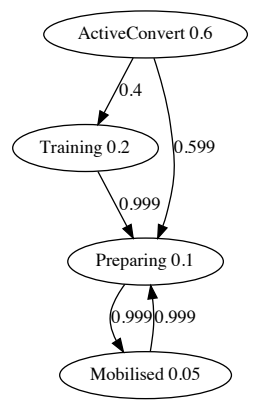

(c) Prior position probabilities

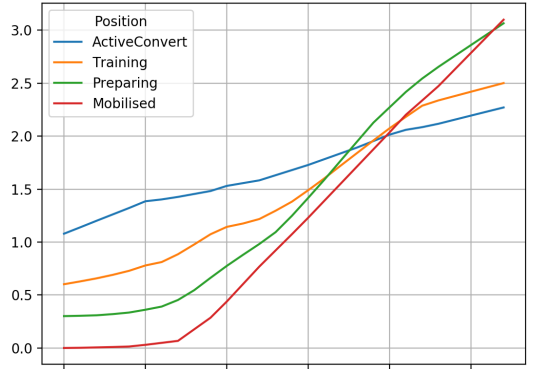

(b) Position score through time

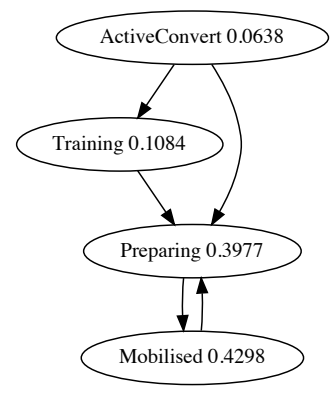

(d) Posterior position probabilities

Figure 6: Results for Scenario A using base RDCEG structure 


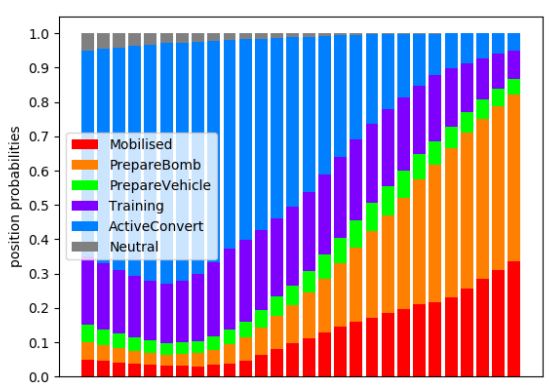

(a) State probabilities through time

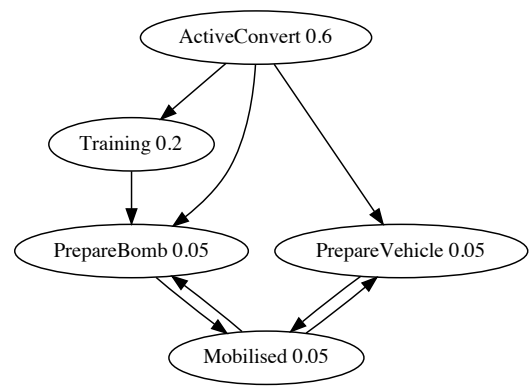

(c) Prior position probabilities

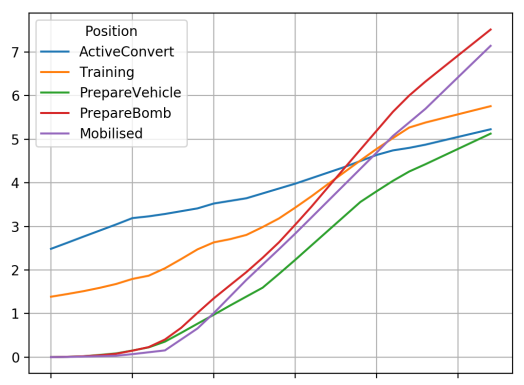

(b) Position score through time

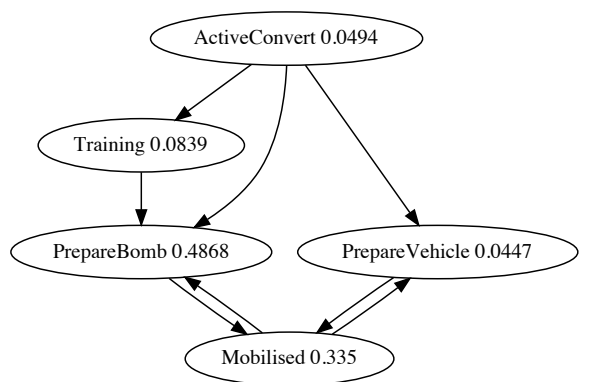

(d) Posterior position probabilities

Figure 7: Results for Scenario A using finer RDCEG structure 


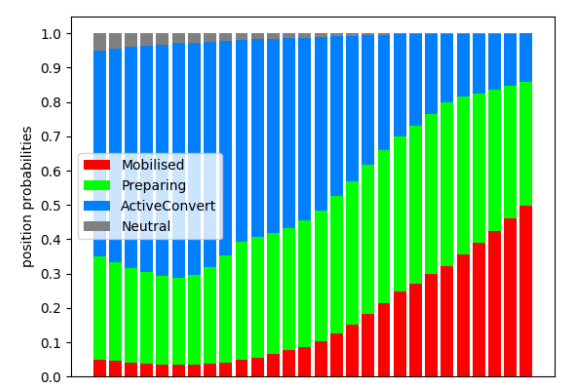

(a) State probabilities through time

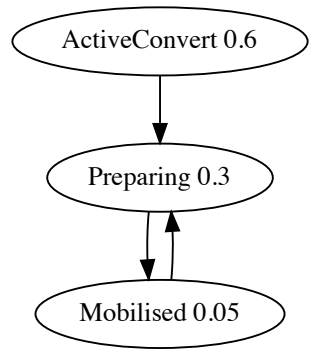

(c) Prior position probabilities

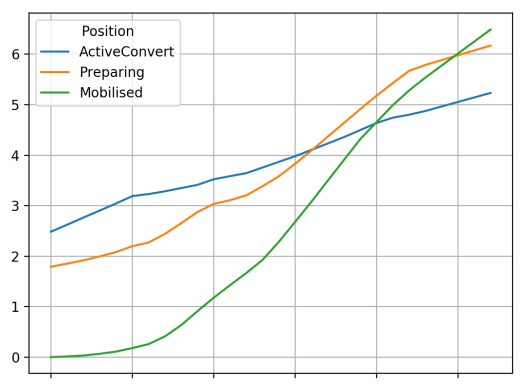

(b) Position score through time

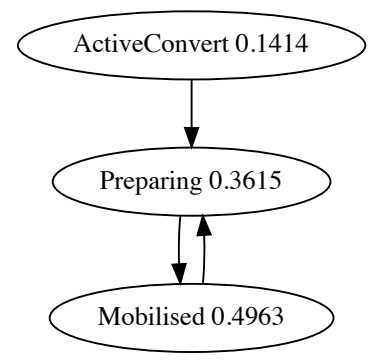

(d) Posterior position probabilities

Figure 8: Results for Scenario A using coarser RDCEG structure 


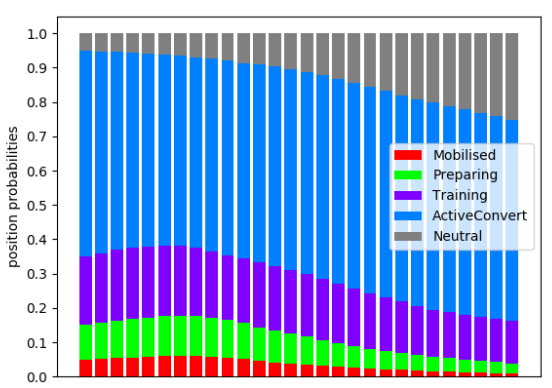

(a) State probabilities through time

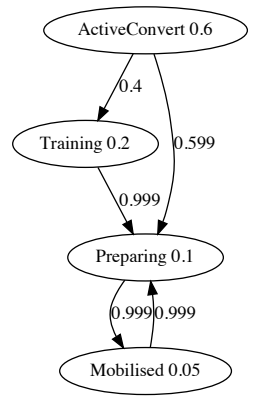

(c) Prior position probabilities

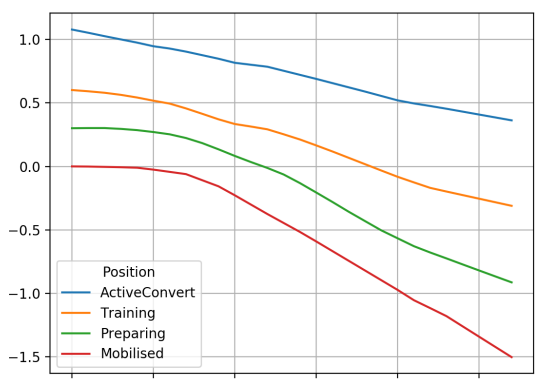

(b) Position score through time

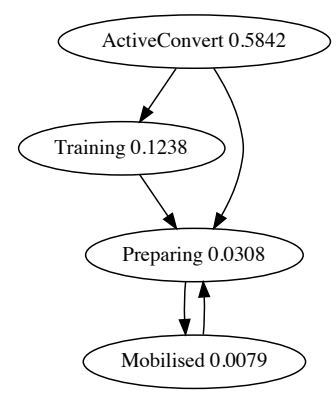

(d) Posterior position probabilities

Figure 9: Results for Scenario B using base RDCEG structure 


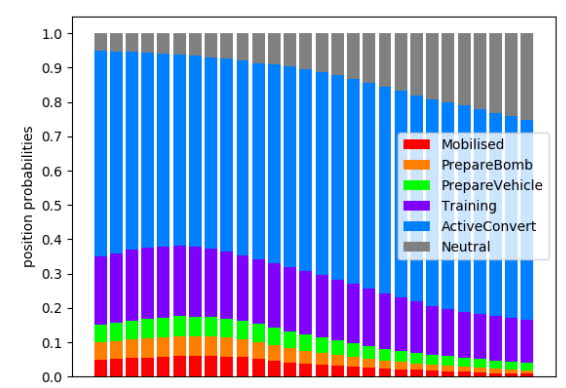

(a) State probabilities through time

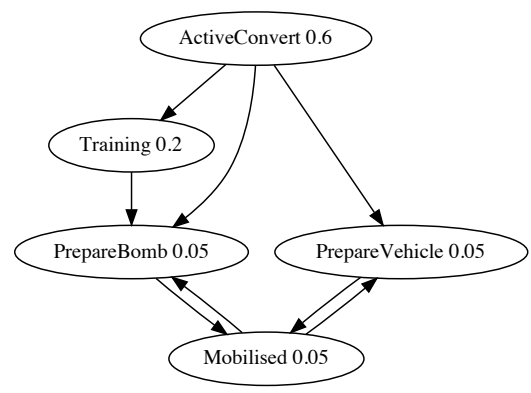

(c) Prior position probabilities

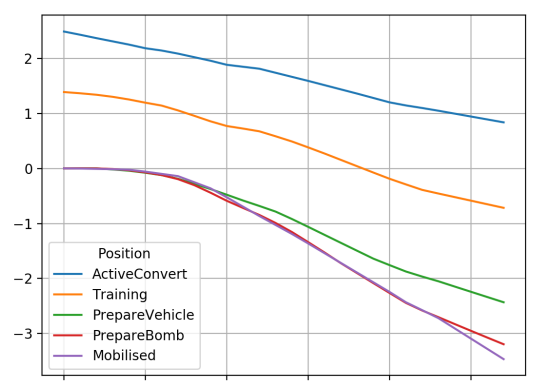

(b) Position score through time

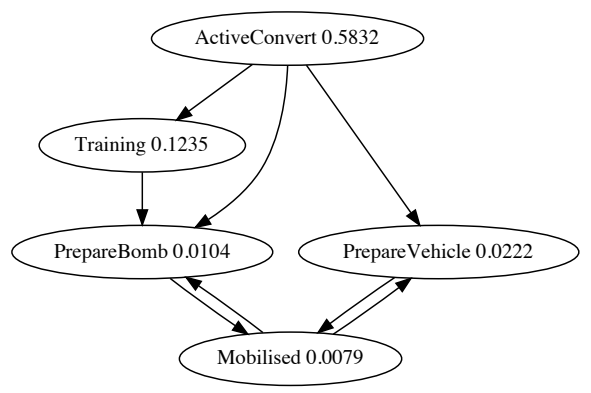

(d) Posterior position probabilities

Figure 10: Results for Scenario B using finer RDCEG structure 


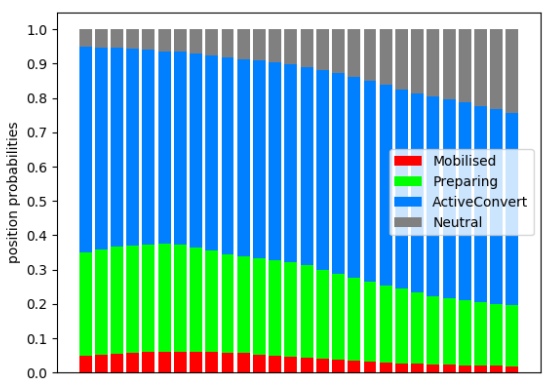

(a) State probabilities through time

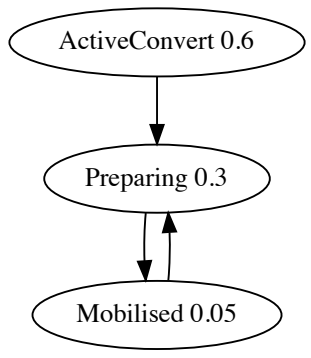

(c) Prior position probabilities

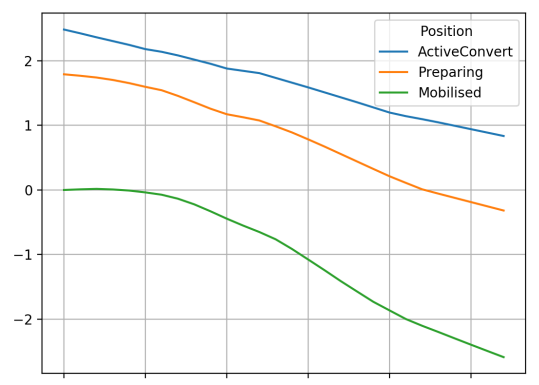

(b) Position score through time

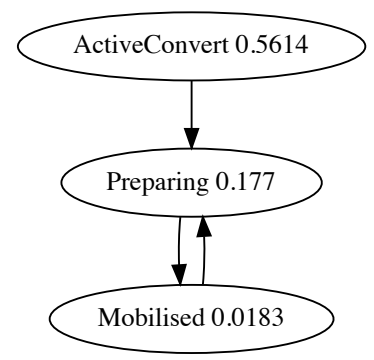

(d) Posterior position probabilities

Figure 11: Results for Scenario B using coarser RDCEG structure 


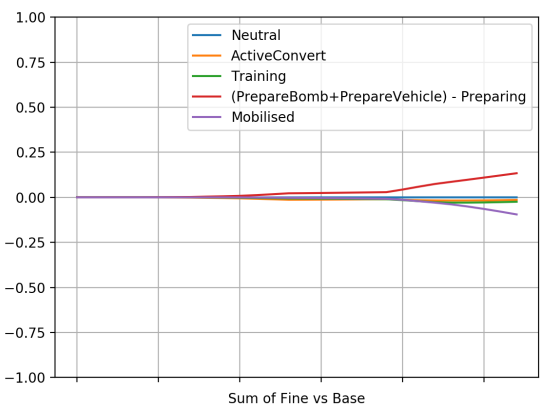

(a) Refined RDCEG minus Base RDCEG state probabilities through time

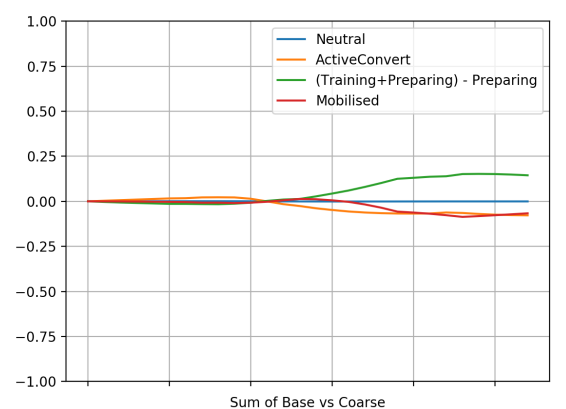

(b) Base RDCEG minus Coarse RDCEG state probabilities through time

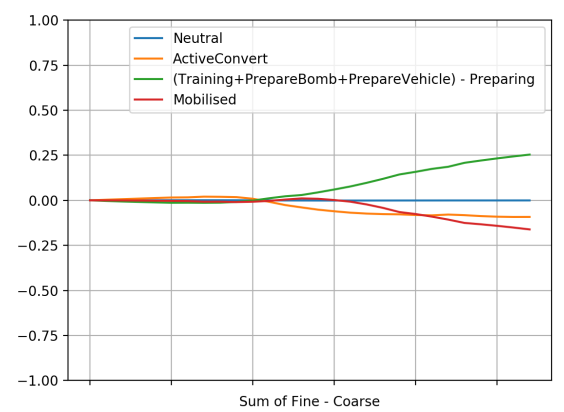

(c) Refined RDCEG minus Coarse RDCEG state probabilities through time

Figure 12: Analysis of state probabilities across RDCEG structure using data from Scenario A 


\section{Appendix E: Sensitivity analysis}

Again we use the model as described in Section 5 as the base RDCEG structure, base prior state probabilities and base holding distribution. We use Scenarios A and B in Appendix $\mathrm{C}$ to analyse the effect on the probability evolution through time under changes in the state prior probabilities and changes in the holding distribution parameter $\zeta$. Figure 13 and Tables 2 and 3 illustrate these impacts. 


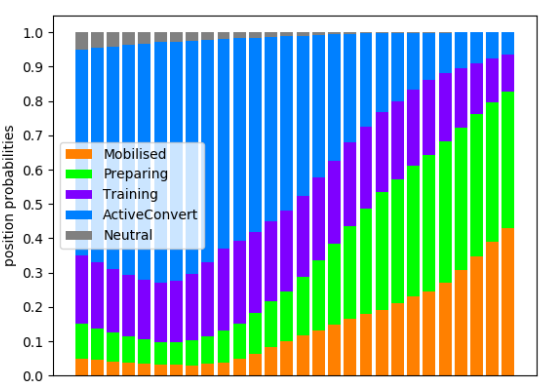

(a) Original prior state probabilities

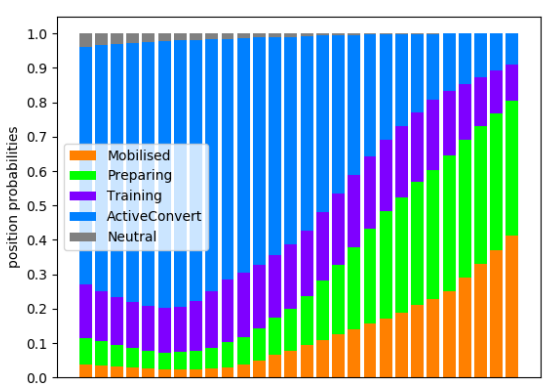

(c) Increasing Active Convert prior by 0.3 then renormalising

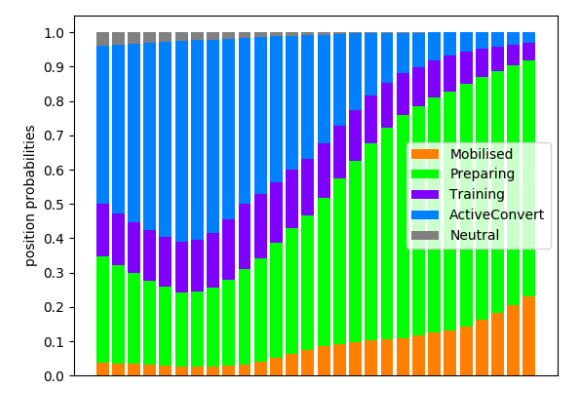

(e) Increasing Preparing prior by 0.3 then renormalising

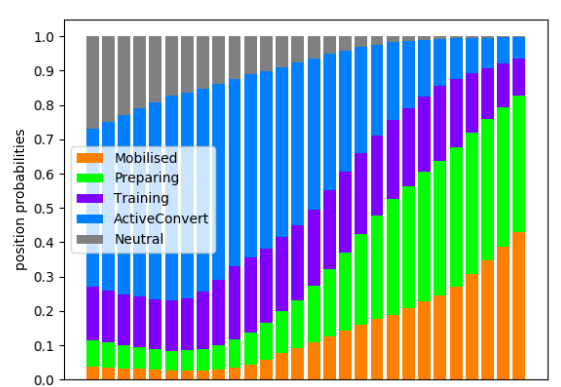

(b) Increasing Neutral prior by 0.3 then renormalising

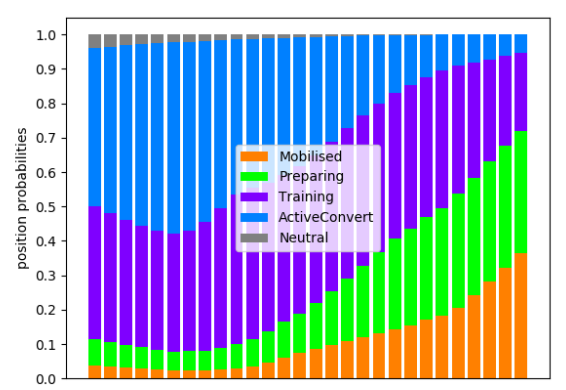

(d) Increasing Training prior by 0.3 then renormalising

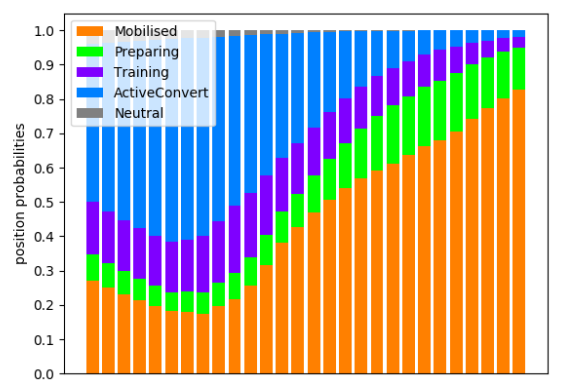

(f) Increasing Mobilised prior by 0.3 then renormalising

Figure 13: Scenario A state prior probability sensitivity analysis: Moderate shifts 


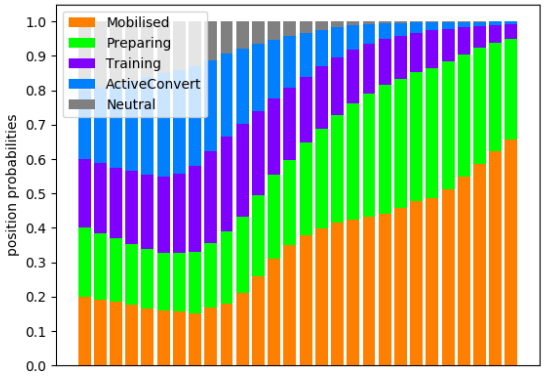

(g) All state priors set equal

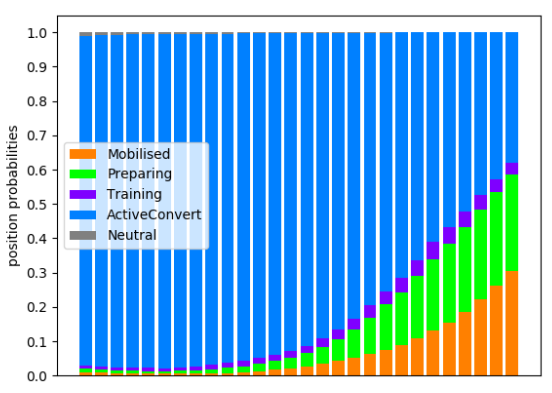

(i) Increasing Active Convert prior to 0.962

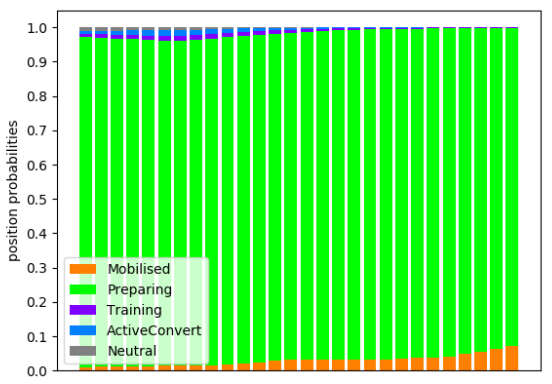

(k) Increasing Preparing prior to 0.962

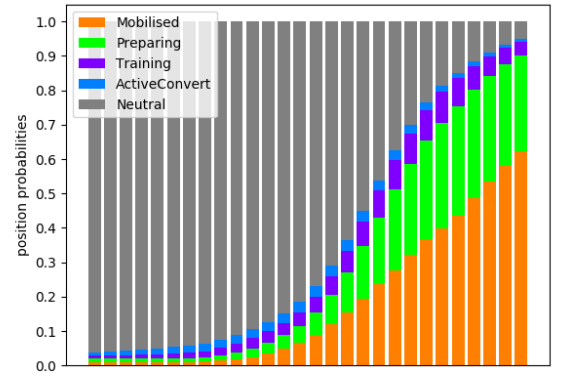

(h) Increasing Neutral prior to 0.962

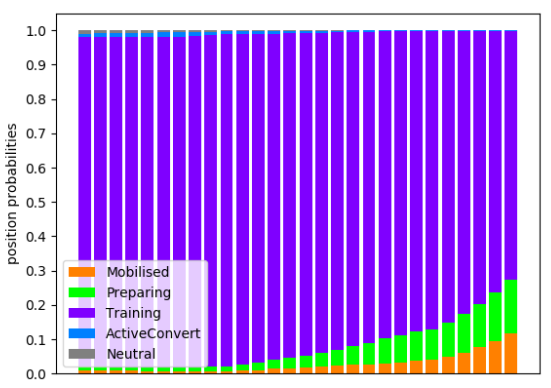

(j) Increasing Training prior to 0.962

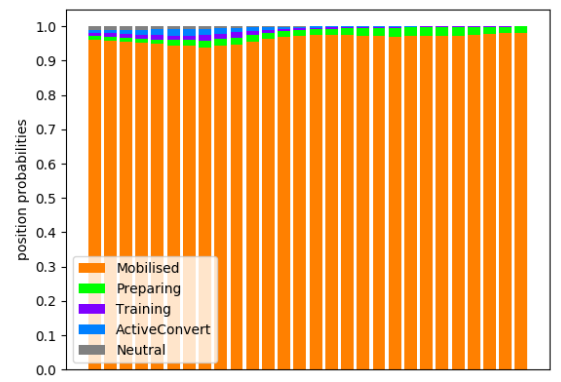

(l) Increasing Mobilised prior to 0.962

Figure 13: Scenario A State prior probability sensitivity analysis: Extreme shifts 


\begin{tabular}{|c|c|c|c|c|c|c|c|}
\hline State & SubScenario & Prior & $\mathrm{t} 5$ & $\mathrm{t} 10$ & $\mathrm{t} 15$ & $\mathrm{t} 20$ & $\mathrm{t} 26$ \\
\hline Neutral & $\mathrm{a}$ & 0.050 & 0.028 & 0.016 & 0.006 & 0.001 & 0.000 \\
\hline ActiveConvert & $\mathrm{a}$ & 0.600 & 0.696 & 0.566 & 0.367 & 0.167 & 0.064 \\
\hline Training & $\mathrm{a}$ & 0.200 & 0.178 & 0.235 & 0.242 & 0.221 & 0.108 \\
\hline Preparing & $\mathrm{a}$ & 0.100 & 0.068 & 0.119 & 0.235 & 0.380 & 0.398 \\
\hline Mobilised & $\mathrm{a}$ & 0.050 & 0.031 & 0.063 & 0.149 & 0.231 & 0.430 \\
\hline Neutral & $\mathrm{b}$ & 0.269 & 0.165 & 0.100 & 0.041 & 0.010 & 0.002 \\
\hline ActiveConvert & $\mathrm{b}$ & 0.462 & 0.597 & 0.518 & 0.354 & 0.165 & 0.064 \\
\hline Training & $\mathrm{b}$ & 0.154 & 0.153 & 0.215 & 0.234 & 0.219 & 0.108 \\
\hline Preparing & $\mathrm{b}$ & 0.077 & 0.058 & 0.109 & 0.227 & 0.377 & 0.397 \\
\hline Mobilised & $\mathrm{b}$ & 0.038 & 0.026 & 0.058 & 0.144 & 0.229 & 0.429 \\
\hline Neutral & $\mathrm{c}$ & 0.038 & 0.020 & 0.012 & 0.005 & 0.001 & 0.000 \\
\hline ActiveConvert & $\mathrm{c}$ & 0.692 & 0.773 & 0.660 & 0.462 & 0.228 & 0.091 \\
\hline Training & $\mathrm{c}$ & 0.154 & 0.133 & 0.184 & 0.205 & 0.203 & 0.105 \\
\hline Preparing & $\mathrm{c}$ & 0.077 & 0.051 & 0.095 & 0.203 & 0.357 & 0.392 \\
\hline Mobilised & $\mathrm{c}$ & 0.038 & 0.023 & 0.049 & 0.125 & 0.211 & 0.411 \\
\hline Neutral & d & 0.038 & 0.022 & 0.012 & 0.004 & 0.001 & 0.000 \\
\hline ActiveConvert & d & 0.462 & 0.549 & 0.419 & 0.268 & 0.124 & 0.054 \\
\hline Training & d & 0.385 & 0.350 & 0.431 & 0.438 & 0.405 & 0.226 \\
\hline Preparing & d & 0.077 & 0.055 & 0.092 & 0.180 & 0.298 & 0.356 \\
\hline Mobilised & $\mathrm{d}$ & 0.038 & 0.024 & 0.047 & 0.109 & 0.172 & 0.364 \\
\hline Neutral & e & 0.038 & 0.023 & 0.012 & 0.004 & 0.001 & 0.000 \\
\hline ActiveConvert & e & 0.462 & 0.582 & 0.425 & 0.223 & 0.082 & 0.030 \\
\hline Training & e & 0.154 & 0.149 & 0.177 & 0.147 & 0.108 & 0.051 \\
\hline Preparing & e & 0.308 & 0.219 & 0.336 & 0.528 & 0.684 & 0.688 \\
\hline Mobilised & $\mathrm{e}$ & 0.038 & 0.027 & 0.051 & 0.098 & 0.126 & 0.231 \\
\hline Neutral & $\mathrm{f}$ & 0.038 & 0.023 & 0.011 & 0.003 & 0.001 & 0.000 \\
\hline ActiveConvert & $\mathrm{f}$ & 0.462 & 0.588 & 0.412 & 0.196 & 0.071 & 0.018 \\
\hline Training & $\mathrm{f}$ & 0.154 & 0.151 & 0.171 & 0.129 & 0.094 & 0.031 \\
\hline Preparing & $\mathrm{f}$ & 0.077 & 0.058 & 0.089 & 0.131 & 0.171 & 0.124 \\
\hline Mobilised & $\mathrm{f}$ & 0.269 & 0.180 & 0.315 & 0.541 & 0.664 & 0.827 \\
\hline
\end{tabular}

Table 2: Posterior state probabilities over time, shown roughly every fifth time period (i.e. every 5 weeks), for moderate shifts in prior state probabilities 


\begin{tabular}{|c|c|c|c|c|c|c|c|}
\hline State & SubScenario & Prior & $\mathrm{t} 5$ & $\mathrm{t} 10$ & $\mathrm{t} 15$ & $\mathrm{t} 20$ & t26 \\
\hline Neutral & $\mathrm{g}$ & 0.200 & 0.142 & 0.065 & 0.017 & 0.003 & 0.001 \\
\hline ActiveConvert & $\mathrm{g}$ & 0.200 & 0.300 & 0.197 & 0.086 & 0.029 & 0.008 \\
\hline Training & $\mathrm{g}$ & 0.200 & 0.229 & 0.243 & 0.169 & 0.115 & 0.042 \\
\hline Preparing & $\mathrm{g}$ & 0.200 & 0.170 & 0.236 & 0.313 & 0.376 & 0.293 \\
\hline Mobilised & $\mathrm{g}$ & 0.200 & 0.158 & 0.260 & 0.414 & 0.477 & 0.656 \\
\hline Neutral & $\mathrm{h}$ & 0.962 & 0.943 & 0.875 & 0.635 & 0.234 & 0.050 \\
\hline ActiveConvert & $\mathrm{h}$ & 0.010 & 0.020 & 0.026 & 0.032 & 0.022 & 0.008 \\
\hline Training & $\mathrm{h}$ & 0.010 & 0.015 & 0.032 & 0.063 & 0.088 & 0.040 \\
\hline Preparing & $\mathrm{h}$ & 0.010 & 0.011 & 0.032 & 0.116 & 0.289 & 0.279 \\
\hline Mobilised & $\mathrm{h}$ & 0.010 & 0.010 & 0.035 & 0.154 & 0.366 & 0.623 \\
\hline Neutral & i & 0.010 & 0.005 & 0.003 & 0.002 & 0.001 & 0.000 \\
\hline ActiveConvert & $\mathrm{i}$ & 0.962 & 0.972 & 0.945 & 0.864 & 0.665 & 0.379 \\
\hline Training & $\mathrm{i}$ & 0.010 & 0.010 & 0.017 & 0.028 & 0.044 & 0.033 \\
\hline Preparing & $\mathrm{i}$ & 0.010 & 0.008 & 0.021 & 0.065 & 0.180 & 0.283 \\
\hline Mobilised & $\mathrm{i}$ & 0.010 & 0.005 & 0.013 & 0.042 & 0.110 & 0.304 \\
\hline Neutral & $\mathrm{j}$ & 0.010 & 0.006 & 0.003 & 0.001 & 0.000 & 0.000 \\
\hline ActiveConvert & $\mathrm{j}$ & 0.010 & 0.013 & 0.008 & 0.005 & 0.002 & 0.001 \\
\hline Training & $\mathrm{j}$ & 0.962 & 0.963 & 0.958 & 0.925 & 0.876 & 0.726 \\
\hline Preparing & $\mathrm{j}$ & 0.010 & 0.012 & 0.022 & 0.047 & 0.084 & 0.155 \\
\hline Mobilised & $\mathrm{j}$ & 0.010 & 0.007 & 0.010 & 0.023 & 0.037 & 0.118 \\
\hline Neutral & $\mathrm{k}$ & 0.010 & 0.008 & 0.003 & 0.001 & 0.000 & 0.000 \\
\hline ActiveConvert & $\mathrm{k}$ & 0.010 & 0.017 & 0.008 & 0.003 & 0.001 & 0.000 \\
\hline Training & $\mathrm{k}$ & 0.010 & 0.013 & 0.010 & 0.005 & 0.003 & 0.001 \\
\hline Preparing & $\mathrm{k}$ & 0.962 & 0.948 & 0.956 & 0.958 & 0.959 & 0.927 \\
\hline Mobilised & $\mathrm{k}$ & 0.010 & 0.014 & 0.023 & 0.033 & 0.037 & 0.072 \\
\hline Neutral & 1 & 0.010 & 0.009 & 0.002 & 0.000 & 0.000 & 0.000 \\
\hline ActiveConvert & 1 & 0.010 & 0.018 & 0.007 & 0.002 & 0.001 & 0.000 \\
\hline Training & 1 & 0.010 & 0.014 & 0.009 & 0.004 & 0.002 & 0.001 \\
\hline Preparing & 1 & 0.010 & 0.016 & 0.018 & 0.020 & 0.025 & 0.018 \\
\hline Mobilised & 1 & 0.962 & 0.944 & 0.963 & 0.974 & 0.972 & 0.981 \\
\hline
\end{tabular}

Table 3: Posterior state probabilities over time, shown roughly every fifth time period for i) all state priors set equal to 0.2 ii) each state prior set to 0.962 and the other states set close to 0.01 (rounded to three decimal places) to test behaviour with extreme priors 


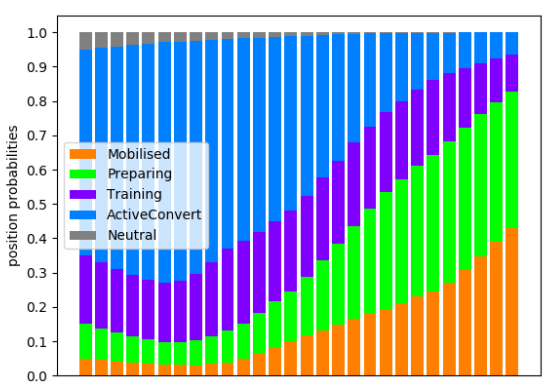

(a) $\zeta=0.001$ as used in base scenario

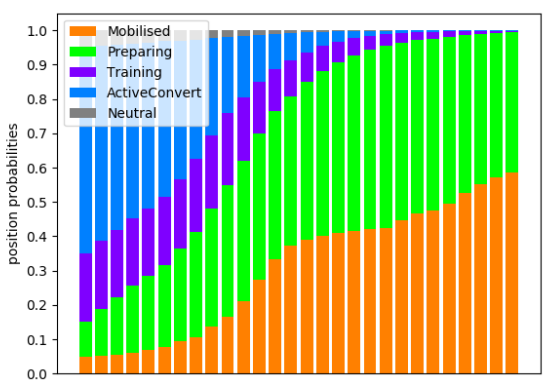

(c) $\zeta=0.1$

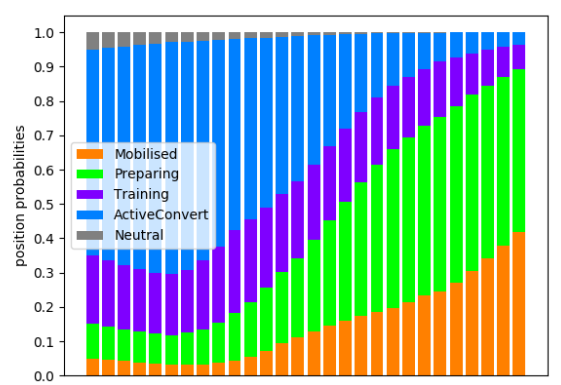

(b) $\zeta=0.01$

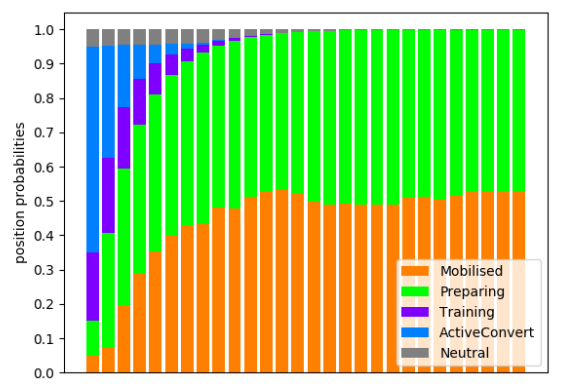

(d) $\zeta=0.5$

Figure 14: Effect on Scenario A posterior probabilities through time of varying the holding time distribution $\zeta$ 


\begin{tabular}{lllllllll}
\hline & State & Prior & $\mathrm{t} 0$ & $\mathrm{t} 5$ & $\mathrm{t} 10$ & $\mathrm{t} 15$ & $\mathrm{t} 20$ & $\mathrm{t} 26$ \\
$\zeta$ & & & & & & & & \\
\hline 0.001 & Neutral & 0.05 & 0.045 & 0.028 & 0.016 & 0.006 & 0.001 & 0.000 \\
& ActiveConvert & 0.6 & 0.625 & 0.696 & 0.566 & 0.367 & 0.167 & 0.064 \\
& Training & 0.2 & 0.193 & 0.178 & 0.235 & 0.242 & 0.221 & 0.108 \\
& Preparing & 0.1 & 0.091 & 0.068 & 0.119 & 0.235 & 0.380 & 0.398 \\
& Mobilised & 0.05 & 0.046 & 0.031 & 0.063 & 0.149 & 0.231 & 0.430 \\
$\zeta$ & State & Prior & $\mathrm{t} 0$ & $\mathrm{t} 5$ & $\mathrm{t} 10$ & $\mathrm{t} 15$ & $\mathrm{t} 20$ & $\mathrm{t} 26$ \\
0.01 & Neutral & 0.05 & 0.045 & 0.028 & 0.015 & 0.005 & 0.001 & 0.000 \\
& ActiveConvert & 0.6 & 0.620 & 0.664 & 0.495 & 0.275 & 0.105 & 0.035 \\
& Training & 0.2 & 0.193 & 0.183 & 0.233 & 0.213 & 0.166 & 0.072 \\
& Preparing & 0.1 & 0.096 & 0.092 & 0.184 & 0.346 & 0.495 & 0.475 \\
& Mobilised & 0.05 & 0.046 & 0.033 & 0.072 & 0.161 & 0.233 & 0.417 \\
$\zeta$ & State & Prior & $\mathrm{t} 0$ & $\mathrm{t} 5$ & $\mathrm{t} 10$ & $\mathrm{t} 15$ & $\mathrm{t} 20$ & $\mathrm{t} 26$ \\
0.1 & Neutral & 0.05 & 0.046 & 0.030 & 0.013 & 0.003 & 0.001 & 0.000 \\
& ActiveConvert & 0.6 & 0.567 & 0.404 & 0.138 & 0.030 & 0.005 & 0.001 \\
& Training & 0.2 & 0.198 & 0.201 & 0.150 & 0.060 & 0.023 & 0.005 \\
& Preparing & 0.1 & 0.138 & 0.272 & 0.427 & 0.497 & 0.505 & 0.408 \\
& Mobilised & 0.05 & 0.050 & 0.093 & 0.273 & 0.410 & 0.466 & 0.587 \\
$\zeta$ & State & Prior & $\mathrm{t} 0$ & $\mathrm{t} 5$ & $\mathrm{t} 10$ & $\mathrm{t} 15$ & $\mathrm{t} 20$ & $\mathrm{t} 26$ \\
0.5 & Neutral & 0.05 & 0.048 & 0.041 & 0.014 & 0.003 & 0.001 & 0.001 \\
& ActiveConvert & 0.6 & 0.326 & 0.015 & 0.000 & 0.000 & 0.000 & 0.000 \\
& Training & 0.2 & 0.221 & 0.037 & 0.001 & 0.000 & 0.000 & 0.000 \\
& Preparing & 0.1 & 0.335 & 0.477 & 0.458 & 0.504 & 0.488 & 0.468 \\
& Mobilised & 0.05 & 0.071 & 0.429 & 0.526 & 0.492 & 0.511 & 0.530 \\
\hline
\end{tabular}

Table 4: Numeric data displayed to 3 decimal places shown for roughly every fifth time step varying the holding time distribution $\zeta$ 